\title{
5. Revision des Invalidenversicherungsgesetzes: Was sie bringen sollte und was nicht
}

Am 5. September haben in der nationalrätlichen Kommission für Soziales und Gesundheit die Beratungen über die 5. IVG-Revision begonnen. Dazu gehörten Hearings, an denen Vertretungen von Behindertenorganisationen, der Invaliden-, Kranken- und Unfallversicherer, der Medizin, der Arbeitgeber, der Gewerkschaften und der Sozialdirektorenkonferenz teilnahmen.

Nachstehend berichte ich über die wesentlichen Gesichtspunkte, die wir aus der Warte der FMH zur Geltung gebracht haben. Zunächst aber einige kurze Hinweise auf die Gliederung der 5. IVG-Revision: Sie hat vorab zum Ziel, durch erhebliche Einsparungen im Laufe der nächsten Jahre zu einer Sanierung des Defizits zu führen, das bei dieser Versicherung beängstigend zunimmt: In den Jahren 2003/2004 betrug es täglich 4,1 Millionen, insgesamt sind es zurzeit etwa 7 Milliarden, über 11 Milliarden dürften es Ende 2007 sein! 20\% Neuberentungen sollen eingespart werden.

Wie soll nun saniert werden? Durch Früherfassungen sollen IV-seitig freiwillige Angebote zum Erhalt der Erwerbsfähigkeit und/oder des Arbeitsplatzes gemacht werden. Angehörige, Verwandte, Arbeitgeber, Versicherer oder Ärzte dürften Betroffene schriftlich bei der IV melden; die Betroffenen selbst würden über diesen Schritt informiert. Wenn die IV-Regionalstelle zum Schluss kommt, dass Frühinterventionsmassnahmen angezeigt sind, fordern sie den Betroffenen auf, sich sofort bei der IV anzumelden. Es folgen dann Eingliederungs- und Integrationsmassnahmen. Ist eine betroffene Person nicht zur Mitwirkung bereit, drohen sanktionierende Schritte. Die Ärzte der regionalen ärztlichen Dienste RAD haben zudem das Recht, von behandelnden Ärzten auch ohne Einwilligung der Betroffenen «notwendige Informationen» einzuholen, und die Ärzte gelten als vom Berufsgeheimnis entbunden. RAD-Ärzte könnten auch beim Arbeitgeber Informationen einholen.

Unsere Kritik setzt an mehreren Stellen an. Dies sind die wichtigsten:

- Das Durchbrechen der ärztlichen Schweigepflicht ist unakzeptabel. Zu Recht wird auch von anderer Seite darauf hingewiesen, dass nicht nur das Vertrauensverhältnis PatientArzt, sondern auch dasjenige zwischen Arbeitnehmer und -geber empfindlich und prognostisch ungünstig gestört würde.
- Früherfassung ist gut, genügt aber nicht. Aus Sicht der Ärzteschaft müsste ein Ausbau der arbeitsmedizinischen Betreuung am Arbeitsplatz anvisiert werden. Ein solcher würde für Arbeitnehmer und Arbeitgeber von Nutzen sein, würde einen echten Dialog und eine daraus resultierende Zusammenarbeit fördern. Ein Job-Coaching, wie es beispielsweise in Bern als Projekt für Psychischkranke praktiziert wird, könnte durchaus zu kostensparenden Synergien beitragen. Verfehlt sind Verknüpfungen von möglichst flächendekkenden Meldungen zur Früherfassung und durchaus sinnvollen Unterstützungsangeboten im Rahmen der Frühintervention mit Zwang und Sanktionsandrohung bei solchen, die eine Mitwirkung verweigern. (Die Idee eines Taggeldversicherungsobligatoriums zur Entlastung der mitarbeitsbereiten Arbeitgeber wurde seitens der Politiker aus Kostengründen in den Wind geschlagen.)

- Eine enge Zusammenarbeit zwischen RADExperten und behandelnden Ärzten ist unabdingbar, da die RAD-Leute lediglich einen momentan geltenden Befund erheben können, während die behandelnden Ärzte die Längsschnittbeobachtung garantieren, die allein massgeblich ist für eine Prognose in Sachen «Erwerbsbehinderung».

- Im Interesse von Kindern und Jugendlichen, die nicht durch Geburtsgebrechen behindert sind, darf auch der Artikel 12 nicht gänzlich gestrichen werden; dadurch sollten alle medizinischen Massnahmen von der Invalidenin die Krankenversicherung übergeführt werden. Was diese Kinder und Jugendlichen brauchen, lässt sich nicht allein unter Heilbehandlungen subsumieren, vielmehr sind häufig Massnahmen im Hinblick auf eine aussichtsreichere Eingliederung nötig. Diese würden in vielen Fällen nicht durch die Krankenkassen bezahlt.

- Dass für Psychischkranke im Verlauf der Erkrankung zum Teil andere Gesetzmässigkeiten gelten als für somatisch Kranke oder Unfallpatienten, stellt für Gesetzgeber und Politiker ein schwerverdauliches Faktum dar. Nichtsdestotrotz setzen wir uns - in Übereinstimmung auch mit Behindertenorganisationen und Vertretern der (Kinder- und Jugend-) Psychiatrie - dafür ein, dass deren Besonder- 
heiten Rechnung getragen wird: Mitwirkungs- und Urteilsfähigkeit können schwanken, zu grosser Druck auf die Rehabilitation kann zu Verschlechterungen oder gar Suizidalität führen, eine Stabilisierung - wie sie dem Gesetzgeber vorschwebt - ist unter Umständen auch nach zwei Jahren Eingliederungsmassnahmen noch nicht erreicht und würde im Fall des Hinausschiebens eines Berentungsentscheides zu sozialen Notlagen führen. Damit verbunden wäre, dass finanzielle Leistungen von der IV zu Gemeinden und Kantonen verlagert würden.

Aus all dem folgt, dass die Arbeitgeber viel stärker einzubinden sind! Nur so können wir uns auch vom Vorwurf befreien, an der Rentenmisere schuld zu sein, nur so können wir unseren fachlich richtigen Standpunkt einbringen. Denn nicht wir, sondern sie tragen die Verantwortung dafür, dass Nischenarbeitsplätze verlorengingen und Zuständigkeiten auf bequeme Art auf die Medizin überwälzt wurden.

Zum Schluss noch ein pikantes Detail: Als wir anfragten, wann denn im Rahmen der Hearings die FMH angehört werden solle, figurierten wir nicht auf der Liste. Es bleibt also noch viel zu tun, bis die Politiker daran denken, die Dachorganisation der Ärzteschaft zu so wichtigen Themen selbstverständlich anzuhören!

Ursula Steiner-König, Vizepräsidentin FMH

\section{$5^{\text {e }}$ révision de la loi sur l'Assurance-invalidité: ce qui est souhaitable et ce qui ne l'est pas}

Le 5 septembre dernier, la Commission du Conseil national pour la sécurité sociale et la santé publique a commencé ses consultations sur la $5^{\mathrm{e}}$ révision de $\mathrm{l}^{\prime} \mathrm{AI}$. Des auditions ont lieu, auxquelles participent des organisations de handicapés, des représentants des assureurs invalidité, maladie et accident, des délégués des milieux médicaux, des employeurs et des syndicats, ainsi que de la Conférence des directeurs des affaires sociales.

J'aborde ci-après les aspects essentiels selon le point de vue de la FMH, tels que nous les avons fait valoir devant la Commission. D'emblée, quelques indications sur la structure de la $5^{\mathrm{e}}$ révision de l'AI: elle a pour premier objectif d'assainir, par de considérables économies au cours des prochaines années, le déficit qui augmente de manière inquiétante dans ce secteur d'assurance. Un déficit qui s'est monté dans les années 2003 à 2004 à 4,1 millions par jour, soit près de 7 milliards aujourd'hui et l'on attend plus de 11 milliards à la fin 2007! Il est prévu que 20\% des nouvelles rentes devront être épargnées.

Comment la révision entend-elle assainir la situation? Par la détection précoce, qui a pour but de la part de l'AI, d'offrir volontairement des possibilités de conserver la capacité ou le poste de travail. Les conjoints et membres de la famille, l'employeur, l'assureur ou les médecins peuvent communiquer le cas par écrit à l'office AI. L'assuré est informé de cette démarche. Lorsque l'office AI parvient à la conclusion que des mesures d'intervention précoces sont indiquées, il invite l'assuré à s'annoncer immédiatement. Des mesures de réinsertion préparant à la réadaptation professionnelle s'ensuivent. Des sanctions peuvent être prononcées si une personne ne participe pas à ces mesures. Les médecins des services médicaux régionaux de l'AI (SMR) ont de surcroît le droit de demander «les informations nécessaires» sans autorisation de l'intéressé, le secret professionnel des médecins étant levé. Il est également proposé que les médecins des SMR puissent demander des informations à l'employeur.

Nos critiques touchent plusieurs points, dont voici les principaux:

- La levée du secret professionnel est inadmissible. A juste titre, on relève aussi dans d'autres milieux que cette démarche toucherait non seulement à la relation de confiance entre médecin et patient, mais également à celle de l'employé et de l'employeur. Les sensibilités et le pronostic en seraient mis à mal. - La détection précoce est une bonne chose, mais ne suffit pas. Du point de vue médical, il faudrait avoir pour objectif l'instauration d'une réelle assistance en médecine du travail sur le lieu de travail de l'employé. Cet aménagement serait utile, tant pour les employés 
que les employeurs, car il créerait un vrai dialogue qui favoriserait la collaboration. Un accompagnement au lieu de travail, comme c'est le cas d'un projet à Berne pour les malades psychiques, pourrait contribuer, par effet de synergie, à des économies de frais. Il est inopportun d'allier une politique d'annonces aussi globales que possible et d'offres de soutien - par ailleurs tout à fait justifiées visant une prévention et une intervention précoces, à des mesures de contrainte et des menaces de sanction à l'encontre de celles et ceux qui refusent de coopérer. (L'idée d'une assurance indemnité journalière obligatoire pour alléger la charge de l'employeur prêt à coopérer a été supprimée par les politiques pour une question de coût.)

- Une étroite collaboration entre les experts des SMR et les médecins traitants est indispensable, les experts des SMR ne pouvant que saisir un état momentané, alors que les médecins traitants bénéficient d'une observation dans la durée, seule à même de garantir un pronostic déterminant pour une «incapacité de travail».

- Dans l'intérêt des enfants et des jeunes gens dont le handicap n'est pas intervenu à la naissance, l'article 12 ne doit pas être abrogé dans sa totalité. Toutes les mesures médicales devraient alors être transférées de l'assurance-invalidité à l'assurance-maladie. Ce dont ont besoin ces enfants et ces adolescents ne saurait consister seulement en soins médicaux, il s'agit bien plus de mesures visant à accroître les chances de (ré-)insertion. Cellesci ne seraient pas, dans de nombreux cas, prises en charge par les caisses-maladie.

- Les modalités légales parfois différentes applicables, en cours de maladie, aux malades psychiques et aux malades somatiques ou aux accidentés, représentent une réalité difficile à accepter pour les législateurs et les politiques. Ce nonobstant, nous nous engageons - avec les organisations de handicapés et les représentants de la pédopsychiatrie pour que ces particularités soient prises en compte: la capacité de jugement et de coopération peut être instable dans de telles situations. Une trop forte pression sur la réadaptation peut conduire à une aggravation du cas et même à une tendance suicidaire, tout comme la stabilisation prônée par le législateur peut ne pas intervenir même après deux ans de mesures de réinsertion. Ceci peut mener, en cas de renvoi d'une décision de rente, à une situation de détresse sociale. De plus, les prestations financières seraient transférées de l'AI aux communes et aux cantons.

Il faudrait, pour conclure, que les employeurs soient beaucoup plus impliqués dans le processus. Ce n'est qu'à cette condition que nous pourrons nous défaire du reproche selon lequel nous serions responsables de la situation catastrophique du payement des rentes. Ce n'est qu'à cette condition que nous pourrons imposer notre point de vue professionnel dûment fondé. Car ce n'est pas à nous de porter la responsabilité de la perte des «emplois de niche», mais bien aux employeurs qui trouvent plus commode de la rejeter sur le monde médical.

Je terminerai par un détail piquant: lorsque nous avons demandé quand la FMH devait être entendue dans le cadre de ces auditions, il s'est trouvé que nous ne figurions pas sur la liste. Il demeure fort à faire pour que les politiques en viennent un jour à consulter spontanément l'organisation faîtière des médecins dans un domaine aussi important que celui-ci!

Ursula Steiner-König, vice-présidente FMH 